\title{
Por el derecho a la educación de la comunidad migrante en Chile: propuestas integrales desde la sociedad civil y diversos actores educativos*
}

\author{
For the right to education of the migrant community in Chile: \\ integrated approaches from the civil society and diverse educational actors
}

\author{
Loreto Jara Males ${ }^{a}$, Eevamaija Vuollo $^{a}$ \\ ${ }^{a}$ Fundación Educación 2020. \\ ljara@educacion2020.cl, evuollo@educacion2020.cl
}

\begin{abstract}
RESUMEN
El actual contexto migratorio en Chile se ha traducido en una serie de desafíos para distintos actores del mundo político, la sociedad civil y, ciertamente, el mundo educativo. Para hacer frente a problemáticas complejas, la convicción de Fundación Educación 2020 ha sido, desde sus orígenes, empoderar a la ciudadanía y fortalecer las capacidades de las comunidades educativas para participar de manera activa en el devenir social. Desde esta perspectiva, 2020 presentó un proyecto que busca fortalecer el derecho a una educación de calidad del estudiantado migrante en Antofagasta, región que ocupa el segundo lugar en la cantidad de población migrante de Chile. El siguiente artículo presenta los resultados parciales de una experiencia de articulación de distintos actores de la sociedad civil para avanzar hacia una educación intercultural y para contribuir al diseño de políticas públicas que consideren la realidad y experiencia del territorio.
\end{abstract}

Palabras claves: Interculturalidad, migración, política pública educativa, Antofagasta, formación docente.

\begin{abstract}
The current context of migration in Chile has resulted in a series of challenges for different actors in the world of politics, civil society and, certainly, in the world of education. In order to face these complex problems, the conviction of a Chilean NGO called Educación 2020 has been, since its origins, to empower the citizenry and strengthen the capacities of the educational communities to participate actively in social development. From this perspective, Educación 2020 presented a project that seeks to strengthen the right to quality education of migrant students in Antofagasta, a region of Chile that has the second highest number of migrant population in Chile. The following article presents the partial results of an experience of articulating different actors of the civil society to advance towards an intercultural education and to contribute to the design of public policies that consider the reality and experience of the territory.
\end{abstract}

Key words: Interculturality, migration, public education policy, Antofagasta, teacher training. 
Estudios Pedagógicos XLV, N 3: 333-351, 2019

POR EL DERECHO A LA EDUCACIÓN DE LA COMUNIDAD MIGRANTE EN CHILE: PROPUESTAS INTEGRALES DESDE LA SOCIEDAD CIVIL Y DIVERSOS ACTORES EDUCATIVOS

\section{INTRODUCCIÓN}

La migración no es un fenómeno reciente ni en el contexto global ni en el contexto chileno, sin embargo, el fenómeno ha tomado nuevas formas durante las últimas décadas, y actualmente hay más gente migrando que nunca en la historia de la humanidad. En América Latina, se ha generado un incremento en el flujo de migración intra-regional a partir de la década de los 90, lo cual ha convertido a Chile a uno de los países más atractivos para los inmigrantes sudamericanos (González en Salas, del Río, Kong \& San Martín, 2016). Según las últimas estimaciones del Instituto Nacional de Estadísticas y el Departamento de Extranjería y Migración, el número de personas extranjeras residentes en Chile llegó a 1.251.225 a fines del año 2018, correspondiendo a un 6,6\% de la población (INE \& DEM, 2019).

El fenómeno migratorio es un hecho relativamente reciente Chile, pero sigue creciendo y ha ido adquiriendo un carácter estructural en varios ámbitos de la sociedad. En el sistema educativo, el estudiantado extranjero representa sólo un 2,2\% de la matrícula total del sistema escolar (Ministerio de Educación, Centro de Estudios, 2018, p. 18), sin embargo, la distribución de estos estudiantes no se ha producido de una manera organizada y balanceada, resultando en guetos en escuelas públicas donde por sus condiciones geográficas y culturales, los porcentajes de inmigrantes pueden ser superiores al $50 \%$ de la matrícula (Jiménez, Valdivia, Morales \& Yáñez, 2017). Considerando que un $60 \%$ de la población extranjera tiene entre 20 y 39 años según las estimaciones de INE y DEM (2019), es probable que la prevalencia de niños y niñas de origen migrante solo seguirá aumentando en las escuelas de Chile.

Varios estudios sobre la inmigración y la discriminación escolar en Chile demuestran que los niños, niñas y jóvenes inmigrantes están expuestos a diversas experiencias de racismo y discriminación de una manera cotidiana en los establecimientos educativos (Riedemann \& Stefoni, 2015). Además, la investigación en este ámbito ha visibilizado bajas expectativas por parte de los docentes hacia los y las estudiantes de origen inmigrante quienes son identificados como "grupos en desventaja o vulnerables", por lo cual los docentes utilizan estrategias compensatorias de calidad inferior o de exigencias mínimas respecto a los objetivos de aprendizaje comunes (Salas et al., 2016), lo que atenta contra una educación de calidad.

La educación no se puede analizar de una manera aislada de la sociedad, y cabe señalar que con el incremento de la inmigración, los prejuicios hacia la población migrante se han hecho visibles en la sociedad chilena más allá del sistema educativo. Una encuesta desarrollada por Espacio Público e Ipsos (2018) sobre la migración e integración social demuestra que un $43 \%$ de los y las encuestadas considera a la migración como dañina para el país porque aumentan los problemas sociales, versus un $53 \%$ que considera la migración como beneficiosa para el país. Por otra parte, la disposición a la integración social es bastante alta, considerando que un $79 \%$ señala que en los barrios de su ciudad debe vivir gente diversa en términos socio-económicos y un $84 \%$ indica lo mismo respecto a los establecimientos educacionales.

Urge promover miradas transversales y sistémicas para abordar las oportunidades y los desafíos de la migración. En Chile, los gobiernos de los últimos años han tenido miradas distintas respecto al tema migratorio, Vargas Rivas y Velasco (2018) plantean en el Informe anual sobre Derechos Humanos en Chile que estas visiones no han respetado, protegido ni promovido los derechos humanos de las personas migrantes en Chile. Además, a pesar 
del incremento en la inmigración hacia Chile, no existen lineamientos gubernamentales respecto al tema (Vargas y Velasco, 2018).

Según un análisis comparativo sobre las regularizaciones migratorias en América Latina realizado por Espacio Público, Chile corresponde al peor país de Latinoamérica en términos de la regularización migratoria y el reconocimiento de derechos de las personas migrantes. El análisis indica que el país presenta incumplimientos respecto al principio de no criminalización, y en términos de legislación chilena, se incumplen estándares como reconocer los derechos de las personas migrantes, y promover el ejercicio de sus derechos y la participación ciudadana (Arís, Meza \& Valenzuela, 2018).

En términos de migración en contextos educativos, existe una Política Nacional de Estudiantes Extranjeros 2018-2022 (Ministerio de Educación, 2018), que tiene por objetivo garantizar el derecho a educación e inclusión de estudiantes extranjeros en el sistema educacional, contribuyendo a fortalecer la calidad educativa, respetando los principios de igualdad y no discriminación enmarcados en los compromisos internacionales firmados y ratificados por el Estado de Chile. Como parte de esta política, la Agencia de la Calidad de la Educación y el Ministerio de Educación desarrollaron distintos espacios de participación con diferentes actores del mundo educativo y, como resultado de ello, dos publicaciones con orientaciones técnicas para la inclusión educativa en contextos migratorios (Ministerio de Educación, 2017a \& 2017b); y la definición de áreas de gestión en las que se necesita actuar para cumplir con el objetivo propuesto por la política: articulación institucional e intersectorial, fortalecimiento de la gestión educativa y vinculación territorial para el diseño de la política educativa.

Más allá de las definiciones planteadas en esta política nacional, así como la serie de demandas que se establecen desde la sociedad civil y que se recogen en el mismo documento (Ministerio de Educación, 2018), las acciones más visibles del gobierno de Chile han estado limitadas a arreglos administrativos que tienen que ver principalmente con el acceso al sistema educativo, tales como reemplazar el Rut 100 con el Identificador Provisorio Escolar (IPE) que permitió a estudiantes migrantes en situación irregular acceder a algunos beneficios que el Rut 100 no permitió, no obstante, como señala María Loreto Mora (2018), este identificador permite el acceso a solo cuatro de los diecisiete beneficios de la Juaneb, dejando a los y las estudiantes en esta condición sin acceso a apoyos que requieren de la obtención del RUN y cédula de identidad.

Asimismo, tal como señala el Instituto Nacional de Derechos Humanos (INDH, 2015), el derecho a la educación no tiene que ver solamente con la incorporación o matrícula en un establecimiento educacional, sino que este derecho es vulnerado también cuando se les niegan a las personas las facilidades para incorporarse a la vida escolar en igualdad de condiciones, o si se les discrimina por ser inmigrantes. Desde esta perspectiva cobra mucha relevancia establecer algunas acciones orientadas a difundir los desafíos de la Política Nacional de Estudiantes Migrantes, lo mismo que acompañar a las comunidades educativas con herramientas que les permitan hacerse cargo de dichos desafíos.

Las acciones de los gobiernos no han sido suficientes para proteger el derecho a la educación de todos los niños, niñas y jóvenes en Chile, ni para reconocer el valor de la diversidad en las comunidades educativas y en la sociedad. Tampoco ha habido suficientes acciones para combatir los estereotipos y preconcepciones que afectan los derechos de los migrantes en la sociedad y en diversos espacios educativos, y muchas escuelas se han quedado bastante solas con el desafío de enfrentar el fenómeno migratorio en los contextos educativos. 
Estudios Pedagógicos XLV, $\mathrm{N}^{\circ}$ 3: 333-351, 2019

POR EL DERECHO A LA EDUCACIÓN DE LA COMUNIDAD MIGRANTE EN CHILE: PROPUESTAS INTEGRALES DESDE LA SOCIEDAD CIVIL Y DIVERSOS ACTORES EDUCATIVOS

Por estas razones, varias organizaciones de la sociedad civil han asumido un rol importante en el tema migratorio y han desarrollado diversos programas para transformar la mirada sobre la inmigración en las escuelas hacia una oportunidad para que las comunidades educativas puedan mirar y repensar su quehacer cotidiano. En el año 2018, la Fundación Educación 2020 empezó a desarrollar un proyecto en la provincia de Antofagasta con el apoyo en la Unión Europea para promover el derecho a la educación de los y las estudiantes inmigrantes, potenciando las respuestas educativas con un enfoque intercultural en comunidades educativas diversas. La convicción de la Fundación ha sido, desde sus orígenes, empoderar a la ciudadanía y fortalecer las capacidades de las comunidades educativas para participar de manera activa en el devenir social, y el proyecto que será presentado en este artículo con sus resultados preliminares, se basa en comprender a la institución escuela como el medio más eficaz para combatir actitudes discriminatorias y para construir una sociedad más inclusiva.

\section{PRESENTACIÓN DEL PROYECTO}

El fenómeno migratorio en el Chile actual, creciente en dimensión y complejidad, no se ha producido de una manera organizada y balanceada. Chile es signatario del Pacto Internacional de Derechos Económicos, Sociales, y Culturales, que en su Artículo 13 "reconoce el derecho de toda persona a una educación", educación que "debe orientarse hacia el pleno desarrollo de la personalidad humana y del sentido de su dignidad, y debe fortalecer el respeto por los derechos humanos y las libertades fundamentales". No obstante, como se mencionó anteriormente, no necesariamente se está cumpliendo con estas obligaciones en la actualidad, lo que puede resultar especialmente relevante en el ámbito del derecho de las migrantes a una educación de calidad, considerando el progresivo aumento de extranjeros en Chile y una legislación insuficiente para abordar la situación.

En efecto, si se considera que el número de estudiantes migrantes se duplicó entre 2015-2016, pasando de 30.625 a 61.086 estudiantes (Ministerio de Educación, 2018), se justifica la necesidad urgente de avanzar en esta materia, y por otro lado, si se considera que proporcionalmente se concentran en la zona norte del país, corresponde actuar focalizadamente desde el punto de vista territorial. En la región de Antofagasta, la proporción de estudiantes extranjeros pasó del 4,5\% en 2015 al 8,4\% en el año 2017, y un $96 \%$ de los distritos censales con establecimientos educativos tienen alumnado extranjero (Ministerio de Educación, Centro de Estudios, 2018). Atendiendo a estas características demográficas, y considerando diversificar los territorios desde el punto de vista urbano/ rural, es que el proyecto de Educación 2020 decide trabajar en tres comunas de la provincia de Antofagasta con el proyecto aMezclarse: más diversidad, mejor educación: Antofagasta, Mejillones y Sierra Gorda.

Este proyecto, financiado por Unión Europea, tiene una duración de dos años y tiene como instituciones asociadas a la Fundación para la Superación de la Pobreza, al Instituto Nacional de Derechos Humanos y a la UNESCO, además de tener alianzas con el Servicio Jesuita a Migrantes, la Oficina Internacional de Migraciones y la Universidad Católica del Norte. El gran desafío del proyecto será detectar creencias, derribar prejuicios y buscar acciones que integren, generen o promuevan espacios inclusivos; considerando que obstaculizar la incorporación o matrícula migrante en un establecimiento educacional, 
negar las facilidades para incorporarse a la vida escolar en igualdad de condiciones o discriminar dentro y fuera del aula; son actos que vulneran el derecho a la educación.

El objetivo global del proyecto es contribuir a mejorar la calidad, equidad, e inclusión de la educación para la comunidad migrante de Chile a través del empoderamiento y participación de ellos y de la sociedad civil de forma que se favorezca la mejora de políticas públicas y normativas asociadas a garantizar el derecho a la educación de la población migrante en Chile. Para lograr este objetivo, el proyecto ha sido diseñado a partir de cuatro líneas de acción que involucran de forma orgánica los distintos actores asociados a la educación de la comunidad migrante, involucrando a:

- Comunas de Antofagasta, Mejillones y Sierra Gorda, atendiendo a la diversidad que existe entre lo urbano y lo rural.

- Comunidades escolares (15 escuelas) de las comunas señaladas.

- Estudiantes de enseñanza media, participantes de un proceso formativo a desarrollar como parte del Laboratorio de Incidencia Política y Social.

- Sociedad civil, autoridades de gobierno y representantes de instituciones del Estado, universidades, familias y otros actores del territorio.

- Ciudadanos y ciudadanas participantes de campañas y levantamiento de propuestas.

Se espera que el proyecto tenga un alcance de 5.000 personas, las que interactuarán de formas diferentes con las distintas líneas de acción. Cabe señalar que el sello de Educación 2020 es su enfoque en la innovación, tanto en la sala de clases como en la visibilización de su trabajo; por tanto, el proyecto contempla el uso de herramientas presenciales (talleres y metodologías participativas) y digitales. A continuación, se presentan las cuatro líneas de trabajo con un nivel de detalle que permita comprender su composición y articulación para la generación de cambios que, desde lo local, aporten la generación de condiciones que mejoren la educación intercultural.

\section{LÍNEAS DE ACCIÓN DE AMEZCLARSE: MÁS DIVERSIDAD, MEJOR EDUCACIÓN}

Considerando que la diversidad en espacios educativos tiene la potencialidad de mejorar los aprendizajes de los y las estudiantes y prepararlos para el mundo más allá de la escuela (OECD, 2010), las y los estudiantes de Chile, con especial énfasis en los estudiantes migrantes, serán los destinatarios del proyecto, toda vez que se beneficiarán de un ambiente escolar y una implementación curricular que respete y valore las identidades culturales. Se pretende conseguir este propósito mediante la articulación de cuatro líneas de acción, líneas que, en su conjunto, están destinadas a lograr cambios sustentables a nivel del núcleo pedagógico, que, de acuerdo con las definiciones de Richard Elmore, corresponde a la interrelación entre estudiante, docente y contenido (Elmore, 2010), y por otro lado, se busca incidir en la generación de políticas públicas ad hoc al contexto migratorio en Chile, relevando la experiencia del territorio.

El proyecto se articula en torno a las siguientes líneas de acción: i. Sensibilización de la ciudadanía, ii. Comunidad Educativa Empoderada, iii. Red Intersectorial por la Educación de Calidad de Estudiantes Migrantes y iv. Laboratorio de Incidencia Política y Social. En términos generales, estas líneas de trabajo funcionan de manera articulada, en algunos casos 
con componentes que se desarrollan de forma paralela y en otros casos concatenadamente. En cualquier caso, se busca incidir en el fenómeno de una manera integral, que aborde las complejidades de la educación intercultural y que potencie la participación de las y los ciudadanos desde distintos espacios y de acuerdo a los diferentes roles que juegan en el ámbito educacional.

\subsection{LÍNEA I: SENSIBILIZACIÓN DE LA CIUDADANÍA}

Desde la experiencia de ser sociedad civil, Educación 2020 tiene la convicción de que, para promover el derecho a la educación, se requiere sensibilizar a la ciudadanía y a las autoridades en torno a materias específicas que pueden resultar de difícil comprensión, "explicar en fácil temas técnicos sobre educación y así promoverlos en la agenda de medios y a través de las redes sociales" (Buccioni \& Waissbluth, 2018). En sintonía con estos preceptos, la primera línea de acción del proyecto tiene como objetivo sensibilizar a la ciudadanía y autoridades en relación a la situación de la protección y promoción de los derechos humanos de los migrantes en el sistema escolar. Se desarrolla fundamentalmente en base a acciones comunicacionales orientadas a facilitar a los niños, niñas, jóvenes migrantes y sus familias, el ejercicio y demanda de sus derechos en educación. Por otro lado, se busca sensibilizar y reflexionar en conjunto con la ciudadanía respecto del derecho a una educación de calidad y del valor de la diversidad y la inclusión en contextos de aprendizaje caracterizados por una alta migración.

En otro subcomponente de la línea de trabajo, se llamará a la participación e incidencia ciudadana mediante el levantamiento de propuestas que apunten a considerar las realidades y necesidades de estudiantes extranjeros en los procesos pedagógicos que se llevan a cabo en las escuelas chilenas y/o en los procesos de gestión que se llevan a cabo en el sistema educativo, de modo de avanzar en educación inclusiva con foco intercultural. Para conseguir este nivel de participación y levantamiento colectivo de ideas y propuestas, se trabajará mediante una plataforma interactiva, información que se complementará con espacios de conversación realizados en las líneas II y III del proyecto.

Algunas de las ideas clave que están a la base de la línea Sensibilización de la ciudadanía, son las siguientes:

- La comunidad migrante es bienvenida en Chile.

- Las y los migrantes tienen derecho a una educación de calidad como cualquier niño, niña y joven chileno.

- Las y los migrantes pueden y deben participar en las instancias de creación de políticas públicas.

- Existen diversas experiencias de inclusión educativa y educación intercultural que se desarrollan en escuelas de Antofagasta y el resto de Chile, buenas prácticas que corresponde socializar y fortalecer.

- La comunidad educativa, y en particular los estudiantes, pueden aportar en la erradicación de mitos en torno a los y las migrantes.

- La diversidad contribuye a la generación de mejores aprendizajes.

Las ideas clave señaladas corresponden tanto a la experiencia práctica de Educación 2020, a instrumentos normativos, convenios y directrices internacionales como los de 
la UNESCO (2006), como a las aportaciones teóricas con las que diversos autores han aportado a la reflexión sobre el derecho a la educación, la calidad de la educación, la inclusión educativa y la educación intercultural (Stefoni, Stang \& Riedemann, 2016; Mora, 2018; PRIEM \& FUSUPO, 2017).

\subsection{LÍNEA II: COMUNIDAD EDUCATIVA EMPODERADA}

Generar capacidades y herramientas en las comunidades educativas para asegurar el derecho de las y los estudiantes migrantes a una educación de calidad es el objetivo de esta línea de acción. Consiste en diseñar, ejecutar y coordinar distintas actividades con la comunidad escolar referidas a prácticas y herramientas que permitan promover espacios de educación intercultural y de protección de los derechos humanos en distintas comunidades educativas de tres comunas de la provincia de Antofagasta.

El trabajo con las comunidades educativas se divide en dos componentes: el primero corresponde a las Mesas de Trabajo Comunitario, desarrolladas durante el primer año del proyecto, para generar un levantamiento de ideas, desafíos, propuestas y necesidades que tienen las comunidades educativas en torno a la educación intercultural en la provincia de Antofagasta. Desde el diseño original, se consideró desarrollar 4 sesiones de trabajo en las que se convocara a distintos representantes de las comunidades educativas participantes del proyecto a reflexionar en torno a temáticas específicas vinculadas con diversidad, migración, educación de calidad. Los resultados de este componente, así como el modo en que se configuraron las mesas de trabajo, constituyen la segunda parte de este artículo, en consideración a que constituyen el primer hallazgo preliminar el torno a la generación de políticas públicas para el fortalecimiento del derecho a una educación de calidad para estudiantes migrantes en la provincia de Antofagasta y, como tal, amerita un nivel de desarrollo mayor.

El segundo componente de la línea Comunidad Educativa Empoderada corresponde a un Ciclo de Formación e Intercambio de Experiencias que, en formato de talleres prácticos, permitirán a los diversos profesionales que interactúan con los estudiantes, contar con mayores herramientas para el abordaje de la diversidad en el aula. El diseño de este ciclo de formación responde a los resultados del componente Mesas de Trabajo Comunitario, toda vez que, para la definición de las temáticas, focos y metodologías, se consideran los resultados de las instancias de encuentro y reflexión a que se hacía alusión anteriormente, y a las que se volverá en el apartado siguiente.

\subsection{LÍNEA III: RED INTERSECTORIAL POR LA EDUCACIÓN DE CALIDAD DE ESTUDIANTES MIGRANTES (RIECEM)}

La tercera línea de acción tiene por objetivo establecer una red intersectorial que contribuya al aseguramiento del derecho a la educación y el reconocimiento de los derechos del niño a nivel regional y nacional a través de una institucionalidad provincial. La responsabilidad esencial de esta red será la generación y/o ajuste de normativas, basándose en los diagnósticos y propuestas que van surgiendo de las Mesas de Trabajo Comunitario, así como también de las opiniones que la ciudadanía levante mediante las campañas de sensibilización e incidencia que se realizarán en el transcurso del proyecto; de forma que el trabajo realizado por la ciudadanía se materialice en acciones concretas. A su vez, establecerá las relaciones políticas que favorezcan el respaldo y difusión de los documentos que emanen de su trabajo. 
Estudios Pedagógicos XLV, N 3: 333-351, 2019

POR EL DERECHO A LA EDUCACIÓN DE LA COMUNIDAD MIGRANTE EN CHILE: PROPUESTAS INTEGRALES DESDE LA SOCIEDAD CIVIL Y DIVERSOS ACTORES EDUCATIVOS

Algunas de las acciones que constituyen el quehacer de la Red, corresponden a identificar las distintas instituciones y actores que integran orgánicas de la sociedad civil articuladas en torno a la educación inclusiva e intercultural o a la integración de la población migrante; conformación y/o acople de una red en función de las orgánicas y experiencias previamente instaladas y desarrolladas en el territorio. Corresponderá a la red hacer y/o problematizar un diagnóstico de la situación migrante en la provincia, con especial énfasis en el área educación y considerando los productos que emanen de las mesas de trabajo comunitario. Se espera que durante el segundo año de ejecución del proyecto, la RIECEM centre su análisis y discusión en las propuestas que la ciudadanía ha levantado en la campaña digital de participación, generando propuestas de política pública en educación relacionadas con el resguardo de los derechos de la comunidad migrante y fortaleciendo la incidencia de la población local, así como visibilizando la experiencia del territorio.

\subsection{LÍNEA IV: LABORATORIO DE INCIDENCIA POLÍTICA Y SOCIAL}

Es una instancia que busca fortalecer el ejercicio ciudadano de estudiantes de educación media que provenientes de comunidades educativas caracterizadas por una alta población migrante, para el reconocimiento y promoción de sus derechos. Esta línea se trata de conformar un espacio donde estudiantes, migrantes y chilenos, puedan conocer sus derechos y desarrollar capacidades y herramientas que les permitan protegerlos y exigirlos.

El Laboratorio es un programa de formación teórica y práctica que se desarrolla durante un semestre académico, y consiste en la generación de diversas instancias de trabajo participativo entre estudiantes y facilitadores expertos que, desde distintos ámbitos, abordarán el desarrollo de habilidades comunicacionales y de formulación de proyectos para generar iniciativas a nivel local. Los objetivos del Laboratorio son formar en los y las jóvenes participantes competencias de incidencia ciudadana que permitan el desarrollo de iniciativas escalables, promover el desarrollo de liderazgos como herramientas útiles y relevantes para el desarrollo del país e incorporar herramientas para el diseño, ejecución y evaluación de proyectos piloto relacionados con temáticas de interculturalidad, Derechos Humanos y de género. Al finalizar el Laboratorio, los y las jóvenes presentarán el resultado de sus proyectos en una instancia pública en la que se espera puedan dialogar con autoridades, expertos y distintos profesionales y actores de la educación; compartiendo sus aprendizajes y contribuyendo a la creación de nuevos conocimientos y modos de hacer política pública.

\section{MESAS DE TRABAJO COMUNITARIO: LA VOZ DE LAS COMUNIDADES EDUCATIVAS}

Como se señalaba, un componente central de la línea de acción Comunidad Educativa Empoderada corresponde a las Mesas de Trabajo Comunitario, instancia desarrollada durante el segundo semestre de 2018 y sobre la que es posible reportar resultados, toda vez que ya fueron analizadas y sistematizadas las aportaciones de quienes participaron en estos encuentros. Este análisis constituye el elemento central de este artículo, ya que representa la voz de los actores locales organizados en torno a espacios de conversación que permiten levantar los desafíos que enfrentan distintas comunidades educativas de la provincia de Antofagasta en torno a la educación intercultural en el contexto migratorio ya descrito. 
Las Mesas de Trabajo Comunitario fueron diseñadas como un espacio de conversación para distintos representantes de las comunidades educativas participantes del proyecto, con la finalidad de levantar información relevante respecto de cómo abordan y necesitan abordar procesos pedagógicos vinculados con la gestión de la diversidad, en el aula y otros espacios de aprendizaje, para desde ahí generar propuestas estructurales que permitan mejorar el acceso a una educación de calidad de las y los estudiantes migrados. En el contexto global del proyecto, el rol de las Mesa de Trabajo Comunitario es clave, ya que generarán parte de los insumos que se discutirán la Red Intersectorial por una Educación de Calidad para Estudiantes Migrantes, cuyo propósito final es generar una propuesta de política pública para fortalecer la educación intercultural.

\subsection{METODOLOGÍA Y ACTORES PARTICIPANTES}

Para el desarrollo de las Mesas, previa vinculación y contacto con las autoridades educacionales respectivas, se convocó a representantes de los equipos docentes o directivos de las instituciones educativas que participarían del proyecto. Las instituciones participantes se detallan en el siguiente cuadro:

Cuadro 1. Instituciones educativas participantes del proyecto aMezclarse

\begin{tabular}{|l|l|}
\hline COMUNA & TIPO DE ESTABLECIMIENTO \\
\hline Antofagasta & $\begin{array}{l}4 \text { jardines infantiles administrados por la Junta Nacional de Jardines Infantiles } \\
\text { (JUNJI). } \\
5 \text { escuelas básicas y } 2 \text { liceos públicos, de la Corporación Municipal de Desarrollo } \\
\text { Social (CMDS). } \\
2 \text { escuelas básicas particulares subvencionadas. } \\
1 \text { liceo particular subvencionado. } \\
1 \text { liceo particular. }\end{array}$ \\
\hline Mejillones & $\begin{array}{l}1 \text { escuela básica pública y 1 liceo administrados por el Departamento de } \\
\text { Administración de Educación Municipal (DAEM). }\end{array}$ \\
\hline Sierra Gorda & $\begin{array}{l}1 \text { escuela básica y 1 liceo administrado por el Departamento de Administración } \\
\text { de Educación Municipal (DAEM). }\end{array}$ \\
\hline
\end{tabular}

Las personas que participaron en las mesas son representantes de las comunidades educativas que, tal como se muestra en el Cuadro 1, corresponden a instituciones de distinta dependencia administrativa y de diferentes niveles educativos, desde educación parvularia hasta educación media. En las 4 sesiones de trabajo que constituyeron las Mesas, participaron alrededor de 45 personas, la mayoría de ellas asistiendo a la totalidad de las sesiones convocadas. El perfil de las y los participantes correspondió a educadoras de párvulo, docentes, encargados de convivencia escolar y directivos, quienes aportaron en las conversaciones desde su experticia en los distintos ámbitos de educación, y permitieron nutrir la conversación a partir de los diferentes roles que cumplen en las instituciones educativas en las que trabajan. 
Estudios Pedagógicos XLV, $\mathrm{N}^{\circ}$ 3: 333-351, 2019

POR EL DERECHO A LA EDUCACIÓN DE LA COMUNIDAD MIGRANTE EN CHILE: PROPUESTAS INTEGRALES DESDE LA SOCIEDAD CIVIL Y DIVERSOS ACTORES EDUCATIVOS

Cada una de las sesiones se articulaba en torno a un tema específico, los que se trataban del rol de la infancia y la juventud en la educación, diversidad cultural en contextos educativos, convivencia y espacios educativos, y pedagogía y diversidad. En cuanto a los contenidos y metodología de trabajo de cada sesión, la primera tuvo un carácter más bien introductorio: presentación del proyecto, los participantes, programación de las siguientes instancias, conversaciones grupales simultáneas en torno a una pauta temática. Las sesiones 2, 3 y 4 contemplaron, además, un encuadre y recuento de lo realizado anteriormente, además de un paso adicional a la conversación grupal, correspondiente a levantar desafíos y propuestas en torno al tema analizado.

Cada sesión se organizó en torno a grupos de trabajo que integraron distintos actores de diferentes comunidades educativas, tendiendo a la diversidad en la composición de los grupos, procurando combinar participantes de distintos estamentos y comunidades educativas. Los grupos funcionaron de manera simultánea, en un espacio físico que permitiera instalar mesas y sillas suficientes para que los grupos puedan conversar y desplazarse libremente. Las conversaciones grupales combinaron técnicas de grupo focal y estrategias utilizadas en procesos de facilitación de conversaciones, produciendo información cualitativa que, a partir de un diseño exploratorio, descriptivo y comprensivo, permitiera profundizar en los conocimientos y las experiencias de cada comunidad educativa. Para el análisis y levantamiento de resultados, se tomó en consideración lo expresado por cada grupo de trabajo, las notas y observaciones de los facilitadores y el registro de audio de cada grupo y sesión.

Con los objetivos específicos de cada una de las sesiones, se buscó complementar y facilitar la consecución del objetivo general del componente Mesas de Trabajo Comunitario, a saber, generar un espacio participativo para el levantamiento de ideas, desafíos, propuestas y necesidades que tienen las comunidades educativas en torno a la educación intercultural en la provincia de Antofagasta. En todo el ciclo se propició que fuesen los propios actores educativos quienes generaran la información y las propuestas acorde su experiencia y conocimientos, siendo de gran importancia la facilitación y contextualización en cada una de las sesiones.

\subsection{RESULTADOS DE LAS MESAS DE TRABAJO COMUNITARIO}

Para analizar y presentar los resultados de las conversaciones desarrolladas en el marco de la línea de trabajo Comunidad Educativa Empoderada, se definieron 3 ámbitos de análisis, basados tanto en la definición del Modelo de Calidad de la Gestión Escolar (Agencia de la Calidad de la Educación, 2013) como en los niveles de presentación de resultados de los Diálogos para la Inclusión de Estudiantes Extranjeros (Mineduc, 2018). Los tres ámbitos definidos fueron:

- Institucional y de liderazgo. Corresponde tanto a las prácticas de gestión de centros educativos como a su articulación con el sistema educacional y las políticas educativas.

- Participación y convivencia escolar. Corresponde a la existencia, promoción y/o gestión de espacios y procesos vinculados con la incorporación activa de distintos actores de la comunidad en los procesos educativos, así como a la interrelación existente entre distintos actores de las comunidades educativas. 
- Curricular y pedagógico. Corresponde a las prácticas y procesos vinculados con el aprendizaje de las y los estudiantes y los modos en que equipos docentes y directivos los comprenden y conducen.

A partir de esta delimitación analítica, se presentan los principales resultados de las conversaciones desarrolladas en las Mesas de Trabajo Comunitario, agrupadas en torno a los principales desafíos, y propuestas concretas para abordarlos.

\subsubsection{Desafíos para avanzar en educación intercultural en la provincia de Antofagasta}

a. Ámbito institucional y de liderazgo

- En la actualidad, el concepto diversidad se asocia con grupos y/o identidades que se consideran como no ajustadas a la norma, lo que se traduce en entender la diversidad como problemática.

- En un contexto de ampliación del reconocimiento de derechos, se requiere una comprensión más profunda respecto de la noción derechos/deberes, la profundidad del derecho a la educación y a la participación.

- Docentes y Educadoras de Párvulos requieren tiempo y acompañamiento para destinar a la formación y abordaje técnico y pedagógico de la diversidad, lo que hoy se ve obstaculizado por la multiplicidad de roles y funciones que deben cumplir.

- Escasa articulación entre los Proyectos de Integración Escolar y las dinámicas de trabajo de docentes.

- Actualmente existen obstáculos para el acceso e integración de la población migrante a los sistemas educativos, por tanto, se requiere avanzar en estrategias para la homologación de niveles educativos.

- Existe poca articulación y homogeneidad en la forma de abordar condiciones socioculturales de niños, niñas y jóvenes entre educación y otros entes estatales; lo que se expresa, por ejemplo, en judicializar la condición de estudiantes en condición de vulnerabilidad. Se requiere articular mejor a la escuela con otras instituciones a nivel del territorio (Estado y sociedad civil), para el uso de un lenguaje común y para la generación de soporte a procesos que se desarrollan en las instituciones educativas.

\section{b. Ámbito de Participación y Convivencia Escolar}

- En los distintos estamentos de las comunidades educativas, las brechas generacionales y las diferencias culturales en ocasiones derivan en prejuicios, estereotipos de género, rechazo a la población migrante, dificultades para resolver conflictos y resistencia a los cambios.

- La participación de las y los estudiantes está limitada a espacios vinculados a lo extracurricular, consistiendo casi exclusivamente en actividades tipo academias, talleres, kermesse u otros similares, sin haber mayor participación e involucramiento en el proceso educativo.

- Se plantea la necesidad de involucrar a las familias en los procesos de aprendizaje 
de las y los estudiantes, desde la educación parvularia (donde se presenta un mayor nivel de participación), hasta la educación media. Se requiere valorar la capacidad que tienen las familias para generar iniciativas y aportar a los procesos educativos.

- $\quad$ Respecto de las familias, se visualiza que existe cierta mirada utilitaria que pueden tener las familias sobre el rol formador de la institución educativa, que es preciso superar.

- Falta continuidad en los programas externos de intervención y asesoría sobre participación infantil.

- Se visualiza que se necesita avanzar en actitudes de escucha activa con las infancias y mayor valoración de la figura de las y los estudiantes en el contexto educativo.

\section{c. Ámbito Curricular y Pedagógico}

- Para enriquecer procesos de aprendizaje en contextos multiculturales, se requiere abordar la dimensión idiomática, especialmente en educación parvularia, dado que las diferencias en pronunciación, modismos y forma de nominar y significar las cosas constituyen obstáculos para la generación de aprendizajes de calidad.

- Se requiere avanzar en flexibilización del currículum, adaptando sus componentes a las realidades específicas de cada comunidad educativa y territorio. Se percibe que las estructuras del currículum dificultan las oportunidades o espacios para abordar el aprendizaje desde una perspectiva intercultural.

- Didácticas y proyectos pedagógicos deben considerar contextos de vulnerabilidad y/o vulneración de derechos, considerando las especificidades que conllevan a la hora de generar aprendizajes.

- Se requiere avanzar en disminuir la competitividad en torno al rendimiento académico del estudiantado y promover la colaboración.

\subsubsection{Propuestas para avanzar en una educación intercultural en Antofagasta}

a. Ámbito Institucional y de Liderazgo

- Planificar y abordar de manera interestamental las temáticas vinculadas a la diversidad, atendiendo a las características y necesidades de cada estudiante desde lo pedagógico, social, cultural y actitudinal, lo que se traduce, por ejemplo, en que equipo psicosocial, docentes y encargado de convivencia están articulados y coordinados para abordar determinadas temáticas y situaciones que se presenten en la comunidad.

- Generar instancias de formación para las comunidades educativas en enfoque intercultural, de género y de derechos humanos, que permita la adquisición de herramientas técnicas y pedagógicas para el abordaje de la diversidad y sus múltiples desafíos.

- Reformular programas de estudio del MINEDUC incorporando perspectiva intercultural, de género y de derechos humanos.

- Entregar insumos y materiales a docentes y educadoras como forma de incentivo y gratificación por la labor realizada. 
- Generar espacios de integración entre los distintos niveles educativos y entre comunidades educativas para socializar estrategias en temas de interculturalidad, convivencia escolar u otros temas relacionados.

- Articular en red los apoyos y colaboraciones entre instituciones que intervienen en la temática de diversidad cultural y educación intercultural.

- Avanzar en conformación de redes de escuelas en el territorio.

\section{b. Ámbito de Participación y Convivencia}

- Generar instancias de diálogo, socialización y participación interestamental dentro de las comunidades educativas, promoviendo una sana convivencia entre estamentos.

- Incluir a los estudiantes en la toma de decisiones de cada comunidad escolar.

- Socializar protocolos de convivencia que existen en distintas comunidades educativas, de modo que permita enriquecer la mirada y compartir lineamientos.

- Promover espacios de formación para los equipos de la comunidad educativa en estrategias de participación, fortaleciendo competencias vinculantes a los nuevos desafíos en cuanto a diversidad e interculturalidad.

- Diseñar estrategias que permitan incluir la participación de niños y niñas que tengan dificultades para relacionarse, asignando roles o nuevas formas de participar en las dinámicas del establecimiento (contribuir en la mantención de espacios comunes, generar paneles informativos, etc.).

- Diseñar estrategias de integración para familias y apoderados, ya sea vinculando su experiencia cotidiana con la labor pedagógica (salidas a terreno para visibilizar el trabajo de los adultos de la comunidad y la experiencia de la interculturalidad, por ejemplo), generando mejores mecanismos de información y retroalimentación sobre el avance de sus hijos y desarrollando actividades recreativas.

- Promover espacios de integración en los patios escolares a través de juegos y expresiones artísticas.

- Reconocer y trabajar con las emociones de niñas y niños con foco en el reconocimiento y la aceptación de quienes conviven en los espacios educativos.

- Avanzar en modos de entender la convivencia que superen el foco en lo disciplinario y conductual, fortaleciendo lo participativo, fomentando la participación de todos los actores que componen la comunidad escolar y parvularia, con especial énfasis en la participación estudiantil.

- Promover el intercambio de experiencias a nivel intergeneracional entre los estudiantes y los demás estamentos.

- Incorporar en los manuales de convivencia el respeto a las expresiones culturales de las nuevas identidades que habitan el contexto educativo.

\section{c. Ámbito Curricular y Pedagógico}

- Desarrollar espacios de reflexión pedagógica para analizar las prácticas de enseñanza, incorporar elementos vinculados a la educación intercultural, trabajar de manera interdisciplinaria, incorporar estrategias de trabajo con Proyectos de Integración Escolar, visibilizar elementos relacionados con la transversalidad, 
flexibilizar el currículum y transformar significativamente la participación de los estudiantes.

- Generar procesos de acompañamiento, intercambio de experiencias y contención entre profesores nobeles y experimentados, a propósito de temas como manejo de grupo, comprensión de la vulnerabilidad, educación emocional, conocimiento intergeneracional, perspectiva de género.

- Flexibilizar el currículum y los instrumentos curriculares asociados (programas de estudio), para dar cuenta de la diversidad y permitir abordajes curriculares que den cuenta de lo diverso.

- Generar autoevaluaciones internas con respecto al trabajo pedagógico.

- Mejorar la selección y priorización de contenidos y objetivos de aprendizaje, involucrando a estudiantes en la definición, por ejemplo, de qué libros leer.

- Generar instancias que permitan el levantamiento colectivo de estrategias para incorporar el enfoque intercultural en la educación.

- Promover la creación y articulación de didácticas interculturales, basadas en el quehacer de docentes y educadoras, apoyadas por profesionales especializados en la temática.

- Potenciar el trabajo interdisciplinar entre docentes.

- Incorporar en las clases de docentes y educadoras las experiencias de base de los estudiantes, así como las palabras usadas por estudiantes de otros países o pueblos originarios, para fomentar la integración y el enriquecimiento cultural.

- Articular el trabajo entre duplas psicosociales, el Programa de Integración Escolar y los equipos docentes para elaborar estrategias compartidas para el trabajo con estudiantes que requieran apoyo.

- Generar estrategias lúdicas que propongan una mirada de apoyo mutuo entre estudiantes.

- Generar estrategias pedagógicas diferenciadas para estudiantes que se incorporan al sistema educativo chileno desde otros países.

- Utilizar TICs para la inclusión educativa y la educación intercultural.

- Contextualizar las experiencias de aprendizaje y discursos pedagógicos considerando a los pueblos originarios y a las distintas culturas de Latinoamérica.

\section{ACCIONES PARA AVANZAR HACIA UNA EDUCACIÓN INTERCULTURAL}

Lo que señalan los actores educativos participantes de este proceso de reflexión en torno a una educación de calidad en condiciones de equidad, coincide con lo que plantean otras perspectivas teóricas de la educación intercultural en la actualidad. Stefoni, Stang y Riedemann (2016) nombran las siguientes seis demandas explícitas de la escuela y su articulación con la formación de un proyecto intercultural:

- $\quad$ Necesidad de una política intercultural

- Adecuación curricular ("un currículum chilenizante")

- Conocimiento del currículo de los países de origen de la población migrante

- Apoyo en el proceso de regularización de los alumnos migrantes

- Barreras idiomáticas 
- Incorporación de todos los actores de la comunidad educativa en la implementación de un modelo intercultural.

Por su parte, desde la experiencia práctica, el Servicio Jesuita a Migrantes, con su programa Migración y Escuela, han identificado los siguientes desafíos que enfrentan las escuelas para aproximarse a la educación intercultural:

- Desafíos pedagógicos

- Desafíos administrativos de la escuela

- Desafíos de convivencia escolar

- Desafíos cultural-idiomáticos

- Desafíos en torno a Derechos Humanos (Linares, Collazos \& Roessler, 2018).

La Fundación para la Superación de la Pobreza, en sus orientaciones desde la práctica, definen seis ejes que generan sinergias, crean un marco institucional, pedagógico y comunitario, que permite innovar y adaptarse al exigente $\mathrm{y}$, a la vez, prometedor contexto educativo intercultural:

- Integración inicial

- Convivencia escolar

- Hacia un currículum y pedagogía intercultural

- Participación protagónica

- Colaboración Familia-Escuela

- Dimensión política (Fundación Superación de la Pobreza \& Instituto de Estudios Municipales, 2016).

Como se desprende de lo anteriormente expuesto, avanzar hacia una educación intercultural es una tarea multidimensional, que requiere de la articulación de distintos ámbitos de acción que operan tanto a nivel de sistema educativo en su conjunto, como al interior de cada institución educativa y en las creencias, percepciones y disposiciones del profesorado hacia generar prácticas pedagógicas inclusivas.

Un ámbito de alta relevancia es el de la formación docente: "La evidencia investigativa es coincidente en concluir que la FID resulta deficiente en lo referido a las temáticas de educación intercultural e inclusión de alumnos inmigrantes y se hace necesaria formación inicial y continua" (Mora, 2018). Asimismo, los resultados del cuarto Censo Docente sobre migración demuestran que cuando se les consulta a los docentes con alumnado migrante respecto de su formación inicial, un 53,8\% de ellos indica haberse formado "para trabajar con estudiantes similares costumbres, valores y modos del habla", y solo un 23,9\% señala haber recibido formación para integrar a estudiantes migrantes (Eduglobal \& Fundación Interhumanos, 2018, p. 40).

Tomando en cuenta los antecedentes expuestos, desde Educación 2020, en concordancia con el diseño del Proyecto aMezclarse y las líneas de acción que contempla, se recogerá el análisis de las Mesas de Trabajo Comunitario para diseñar e implementar un ciclo de formación e intercambio de experiencia que se realizará con las comunidades educativas participantes del proyecto durante el segundo año de implementación. Para responder a los desafíos identificados en torno a los tres ámbitos (institucional y liderazgo; participación y 
Estudios Pedagógicos XLV, $\mathrm{N}^{\circ}$ 3: 333-351, 2019

POR EL DERECHO A LA EDUCACIÓN DE LA COMUNIDAD MIGRANTE EN CHILE: PROPUESTAS INTEGRALES DESDE LA SOCIEDAD CIVIL Y DIVERSOS ACTORES EDUCATIVOS

convivencia escolar; pedagógico y curricular), dentro del trabajo de los talleres se crearán productos concretos que ayudarán a las comunidades educativas en avanzar hacia un enfoque intercultural en su quehacer cotidiano.

Durante el primer semestre, el trabajo se enfoca en el ámbito pedagógico y curricular, desarrollando estrategias de innovación pedagógica en contextos diversos. Las personas participantes tienen una oportunidad para diseñar experiencias de Aprendizaje Basado en Proyectos (ABP) con un enfoque intercultural, de Derechos Humanos y con perspectiva de género, en equipos interdisciplinarios que reúnan a personas de distintos establecimientos educativos y con el apoyo de expertos en innovación pedagógica y Derechos Humanos. Esta estrategia pedagógica permite promover la colaboración entre los distintos actores educativos y entre los y las estudiantes participantes en los proyectos, rompiendo las barreras entre distintas asignaturas y con el mundo más allá de la escuela, permitiendo experiencias de aprendizaje significativas tanto para los estudiantes como para los docentes implementando una nueva manera de aprender y enseñar. Además, las personas participantes en los talleres pueden visualizar distintas maneras para flexibilizar el currículum, ya que el diseño de los proyectos interdisciplinarios se desarrolla integrando Objetivos de Aprendizaje de diversas asignaturas, aprovechando de la flexibilidad que permite el currículum actual.

El segundo semestre se enfocará fuertemente en los ámbitos de participación y convivencia e institucionalidad, utilizando las experiencias de las diversas comunidades educativas para crear planes de fortalecimiento de la interculturalidad para los distintos niveles educativos de una manera colaborativa. Se busca generar instancias de diálogo entre los actores educativos que participan en los talleres, y compartir diversos protocolos que se han desarrollado en los distintos establecimientos para enriquecer la mirada y crear algunos lineamientos de manera colaborativa. Se trabajará el concepto de diversidad en las comunidades educativas más allá de la nacionalidad de los estudiantes, utilizando estrategias concretas para empatizar con estudiantes como individuos, reflexionar en torno a prejuicios y para descubrir las oportunidades que provee un enfoque intercultural en la educación.

Además, durante el ciclo de talleres, se motivará a que las personas participantes presenten sus mejores prácticas y diseños pedagógicos en cuanto a las didácticas en interculturalidad e innovación pedagógica, participación ciudadana, y convivencia intercultural, las que se sistematizarán y conformarán un Banco de Buenas Prácticas que serán difundidas en una página web. Este Banco incluirá los diseños de proyectos ABP con enfoque intercultural y los planes de fortalecimiento de la interculturalidad para la educación parvularia, básica y media desarrollados durante los talleres, y permitirá que dichos aprendizajes trasciendan a los establecimientos difundiendo las buenas prácticas desarrolladas en la provincia. Estos aprendizajes pueden servir como una herramienta a nivel provincial, regional e incluso nacional, proveyendo insumos desde el terreno para propuestas de política pública en la materia.

En articulación con las otras líneas de acción del proyecto, se recogerá esta experiencia para rescatar elementos que constituyan propuestas para fortalecer políticas públicas que aborden la educación desde una perspectiva intercultural; interrelacionando la innovación pedagógica con el fortalecimiento la capacidad de incidencia de los actores educativos y de la ciudadanía. 


\section{CONCLUSIONES}

El proceso migratorio en el Chile actual se vive de manera compleja, tanto por las características que adquiere la migración como por las respuestas de la política pública y la legislación que actualmente se discute en el marco de las propuestas y medidas implementadas en temas migratorios por el gobierno de Sebastián Piñera. A pesar de la creciente atención hacia la inmigración tanto a nivel internacional como nacional, las acciones de los gobiernos en Chile no han sido suficientes para reconocer el valor de la diversidad en la sociedad, ni para proteger el derecho a la educación de todos los niños, niñas y jóvenes sin importar su nacionalidad o condición migratoria.

En el actual escenario, el ámbito educacional adquiere una importancia fundamental, tanto por el desafío permanente de generar educación de calidad en condiciones de equidad, como por el desafío emergente de incorporar un enfoque intercultural a los procesos de aprendizaje, una urgencia a la que han tenido que atender con cierto nivel de improvisación los equipos docentes y directivos de distintos contextos educacionales, especialmente en la educación pública. Por lo tanto, se requiere avanzar en generar herramientas, mecanismos y procesos que colaboren con las instituciones educativas en el cumplimiento de su rol.

Avanzar hacia una educación intercultural es una tarea multidimensional, que requiere de propuestas integrales que articulen distintos ámbitos de acción que operan tanto a nivel de sistema educativo en su conjunto, como al interior de cada institución educativa. Las complejidades de los desafíos que vive cada establecimiento educativo son bien variadas dependiendo de su contexto y su trayectoria con una educación intercultural, sin embargo, se pueden concluir en algunos elementos comunes a los que se debe prestar atención para garantizar el derecho a la educación de la comunidad migrante en Chile.

Primero, es esencial entender que, para avanzar hacia una educación intercultural, se requiere de procesos sistémicos, donde la escuela no tiene la responsabilidad exclusiva, sino que se requiere de avanzar en trabajo en red y en articulación con la institucionalidad local. Asimismo, es relevante avanzar en articulación entre distintos niveles educativos y entre profesionales de diversos ámbitos, promoviendo el trabajo colaborativo e interdisciplinario. Las Mesas de Trabajo Comunitario realizados en el marco del proyecto aMezclarse demostraron que la educación parvularia tiene mucho que aportar a la educación básica y media respecto de cómo gestionar la diversidad en los distintos espacios de aprendizaje, considerando lo lúdico como un elemento fundamental en el proceso y la incorporación de las familias; elementos que se desdibujan en el sistema escolar.

Por otro lado, el foco de las acciones debe mantenerse en lograr cambios a nivel del núcleo pedagógico que corresponde a la interrelación entre estudiante, docente y contenido (Elmore, 2010). Por lo tanto, es fundamental velar por las condiciones de trabajo de los equipos docentes y de una gestión curricular que dé espacio a la reflexión, al análisis de las prácticas pedagógicas y a la generación de contenidos y experiencias exitosas por parte de las distintas comunidades educativas. En especial, se requiere avanzar en análisis, reflexión y producción curricular en las unidades educativas, fomentando los espacios de flexibilidad que permite el currículum nacional y que hoy, aparentemente, no están siendo utilizadas como ventanas de oportunidad para la incorporación del enfoque intercultural en los procesos pedagógicos.

Asimismo, se debe reorientar la formación inicial y continua de docentes de manera que puedan desarrollar competencias interculturales y desafiar sus prejuicios y formas de pensar de una manera crítica. A pesar de que este artículo tenga su foco en la educación 
Estudios Pedagógicos XLV, № 3: 333-351, 2019

POR EL DERECHO A LA EDUCACIÓN DE LA COMUNIDAD MIGRANTE EN CHILE: PROPUESTAS INTEGRALES

DESDE LA SOCIEDAD CIVIL Y DIVERSOS ACTORES EDUCATIVOS

en contextos migratorios, es fundamental destacar que la educación intercultural va mucho más allá del estudiantado extranjero. El gran desafío del sistema educativo es el de gestionar pedagógicamente la diversidad para generar aprendizajes de calidad en todos los estudiantes, sin importar su género, nivel socioeconómico, país de origen, condición migratoria o cualquier otra característica o condición. Para lograr esto, se requiere fortalecer el protagonismo del estudiantado en el proceso educativo, otorgando voz a niños, niñas y jóvenes, involucrando activamente a la figura del estudiante en su proceso educativo y ampliando los espacios de participación y toma de decisiones.

Atendiendo a estas consideraciones, es que el proyecto que se ha presentado en este artículo busca fomentar la participación de distintos actores educativos para generar propuestas integrales y cambios que apunten al fortalecimiento del derecho a una educación de calidad para todas y todos los estudiantes de Chile, sin importar su nacionalidad.

\section{REFERENCIAS BIBLIOGRÁFICAS}

Arís, M., Meza, M. J. \& Valenzuela, P. (2018). Estudio de análisis comparativo regional de las regularizaciones migratorias en América Latina: Argentina, Brasil, Colombia, Chile, Ecuador y Perú. Documento de referencia $N^{o} 39$. Santiago de Chile: Espacio Público.

Buccioni, A., Waissbluth, M. (2018). Educación 2020: las claves de un modelo de incidencia efectivo. Serie Ensayos, $N^{o} 1$, Julio 2018. Santiago, Chile.

Eduglobal \& Fundación Interhumanos. (2018). Resultados 4to Censo Docente - Migración: Su impacto en la práctica docente.

Elmore, R. (2010). Mejorando la escuela desde la sala de clases. Santiago: Área de Educación de la Fundación Chile.

Fundación Superación de la Pobreza \& Instituto de Estudios Municipales. (2016). Educación e Interculturalidad en escuelas públicas: Orientaciones desde la práctica.

García, M. (2013). Educación y desigualdad en Chile: avanzando hacia espacios interculturales. Revista Sociedad \& Equidad, 5, 276-292.

Gorski, P. (2008). Good intentions are not enough: a decolonizing intercultural education. Intercultural Education, 19(6), 515-525. doi: 10.1080/14675980802568319.

INDH. (2015). Cartilla informativa: Derecho a la educación de niños, niñas y adolescentes migrantes. Santiago: INDH.

INE. (2019). Segundo Registro de Chilenos en el Exterior: ¿Cuántos son, dónde están y cómo son los chilenos? Santiago de Chile: Instituto Nacional de Estadísticas, Subdirección de Operaciones.

Jiménez, F., Valdivia, M. A., Morales, R. V. y Yáñez, M. H. (2017). Migración y escuela: Análisis documental en torno a la incorporación de inmigrantes al sistema educativo chileno. Psicoperspectivas, 16(1), 105-116. doi: 10.5027.

Linares, K., Collazos, M. \& Roessler, P. (2018). Migración y escuela: Guía de acciones prácticas hacia la interculturalidad. Santiago de Chile: Servicio Jesuita a Migrantes.

Ministerio de Educación, Centro de Estudios. (2018). Mapa del estudiantado extranjero en el sistema escolar chileno (2015-2017). Documento de trabajo $N^{\circ} 12$. Santiago, Chile.

Ministerio de Educación. (2018). Política Nacional de Estudiantes Extranjeros 2018-2022. Santiago, Chile.

(2017a). Diálogos para la inclusión de estudiantes extranjeros: informe de devolución. Santiago de Chile.

. (2017b). Orientaciones técnicas para la Inclusión educativa de estudiantes extranjeros. Santiago de Chile: División de Educación General, Coordinación Nacional de Inclusión y Diversidad. 
Mora, M. L. (2018). Política educativa para migrantes en Chile: un silencio elocuente. Revista Latinoamericana, 49, 231-257.

OECD. (2010). Education Today 2010: the OECD perspective. OECD Publishing.

PRIEM (Programa Interdisciplinario de Estudios Migratorios) \& FUSUPO (Fundación para la Superación de la Pobreza). (2017). Guía pedagógica para una educación intercultural, antiracista y con perspectiva de género: ideas, experiencias y herramientas. Santiago de Chile.

Riedemann, A. \& Stefoni, C. (2015). Sobre el racismo, su negación, y las consecuencias para una educación anti-racista en la enseñanza secundaria chilena. Revista Latinoamericana, 4(42), 191216.

Salas, N., del Río, M.F., Kong, F. \& San Martín, C. (2016). Caracterización y prejuicio acerca de los inmigrantes en el sistema escolar. Universidad Diego Portales: Proyecto FONIDE No 911424.

Stefoni, C., Stang, F. \& Riedemann, A. (2016). Educación e interculturalidad en Chile: un marco para el análisis. Estudios internacionales (Santiago), 48(185), 153-182.

UNESCO. (2006). Directrices de la UNESCO sobre la educación intercultural. París: UNESCO.

Vargas Rivas, F. \& Velasco, J. C. (2018). Derechos de las personas migrantes y refugiadas: cambios en materia migratoria en Chile. En Vial, T. (Editor). Informe anual sobre Derechos Humanos en Chile 2018. Centro de Derechos Humanos UDP. 
